\title{
A genetic and spatial Bayesian analysis of mastitis resistance
}

\author{
Solve $\mathrm{S}_{\text {ÆB }} \emptyset^{\mathrm{a} *}$, Arnoldo FrigeSSI ${ }^{\mathrm{b}}$ \\ ${ }^{a}$ IKBM, Agricultural University of Norway, PO Box 5003, 1432 Ås, Norway \\ ${ }^{\mathrm{b}}$ Section of Medical Statistics, University of Oslo, and Norwegian Computing Center, \\ Oslo, Norway
}

(Received 3 December 2003; accepted 26 April 2004)

\begin{abstract}
A nationwide health card recording system for dairy cattle was introduced in Norway in 1975 (the Norwegian Cattle Health Services). The data base holds information on mastitis occurrences on an individual cow basis. A reduction in mastitis frequency across the population is desired, and for this purpose risk factors are investigated. In this paper a Bayesian proportional hazards model is used for modelling the time to first veterinary treatment of clinical mastitis, including both genetic and environmental covariates. Sire effects were modelled as shared random components, and veterinary district was included as an environmental effect with prior spatial smoothing. A non-informative smoothing prior was assumed for the baseline hazard, and Markov chain Monte Carlo methods (MCMC) were used for inference. We propose a new measure of quality for sires, in terms of their posterior probability of being among the, say $10 \%$ best sires. The probability is an easily interpretable measure that can be directly used to rank sires. Estimating these complex probabilities is straightforward in an MCMC setting. The results indicate considerable differences between sires with regards to their daughters disease resistance. A regional effect was also discovered with the lowest risk of disease in the south-eastern parts of Norway.
\end{abstract}

disease resistance / genetic effect / Markov chain Monte Carlo / spatial smoothing / survival analysis

\section{INTRODUCTION}

Mastitis is an infectious disease causing an inflammation in the mammary glands of dairy cattle. The typical consequence is reduced milk quality and yield. Since mastitis is a frequent disease, the economic loss due to the reduced production can be substantial. Increasing the disease resistance among dairy cattle is therefore desirable.

The pathogens causing mastitis are various species of bacteria, but a cow's susceptibility to the disease also depends on many other factors. It is known

*Corresponding author: solve.sabo@ikbm.nlh.no 
that environmental factors like hygienic conditions, climate and stock size, among others, are influential $[1,17,18]$. In addition the disease susceptibility may be genetically dependent, in which case disease resistance could be improved by animal selection through breeding programmes.

Mastitis in first lactation cows has been studied by several authors, and usually mastitis is treated as a binary occurrence variable $[4,9,10]$. However, due to cows leaving the study for various reasons (random censoring) the data are incomplete, which may lead to biased results if such observations are left out or treated as non-occurrences. This strongly motivates using survival analysis methodology, as we do here, where mastitis resistance is considered as a survival trait. Survival models elegantly handle censored observations.

The effects of both environmental and genetic factors on mastitis resistance were analysed by means of a proportional hazards model. The purpose of this study was twofold: (1) construct an informative criterion for ranking of breeding animals which reflect their genetic potential with respect to mastitis resistance and (2) conduct a geographical analysis of mastitis (within Norway) in order to investigate any spatial patterns of the disease.

The genetic evaluation of breeding animals related to continuous phenotypic values has become routine using linear mixed models, and prediction of animal specific genetic values by means of the best linear unbiased predictor (BLUP) is straight forward [8]. The prediction of random effects using classical methods is, however, more complicated when the response is a binary variable or a censored survival time variable. For such problems adopting a Bayesian approach has proven to be a fruitful strategy $[15,19]$. Not only does the Bayesian approach in conjunction with Markov chain Monte Carlo (MCMC) methods make the analysis feasible, but as we show in this paper, posterior probabilities on the ranking of animals can easily be derived from the MCMC output. We found these probabilities more informative than the mere ranking of animals based on their genetic values which is usually presented.

The environmental variation due to herd effects is not explicitly considered, only at an aggregated veterinary districts level. In the spatial analysis it was assumed that geographically adjacent veterinary districts have similarities with respect to environmental conditions, and a priori dependencies between districts were included. The presented methodology can easily be extended to further hierarchical levels, such as herds, but a full analysis is left to a future study and the results should thus be regarded as "first results".

In Section 2 we suggest a proportional hazards model, with a smooth prior on a piecewise constant baseline hazard. The regression includes a genetic and a spatial effect. The sire effect is modelled with the help of the known 
pedigree. The spatial effect is based on veterinary districts and captures climatic, environmental, herd and veterinary practice factors. Bayesian inference is performed by means of MCMC, as explained in Section 3. In Section 4 we suggest to study posterior rankings of sires, in terms of their effects on mastitis resistance and to individuate the posterior probability of being among the top $10 \%$ sires as a guideline for cattle management. The results are reported in Section 5. We close the paper with a short discussion of the limits and potentials of our approach.

\section{DATA AND MODEL}

The data were extracted from the data set analysed by Heringstad, Klemetsdal and Ruane [9] and included records on $n=36178$ first lactation cows of Norwegian Cattle (NRF). These were daughters of $n_{s}=245$ sires, and the number of daughters per sire ranged from 22 to 205. On average, the sires had daughters in 66 veterinary districts. For each cow the mastitis resistance was measured as the number of days from day 31 before first calving to first veterinary treatment of clinical mastitis. The cows were dried off (milking was stopped) about 60 days before an upcoming calving, and the risk of mastitis is expected to drop in the dry-period. As the mammary glands prepare for a new lactation, the cows may have mastitis even prepartum, and it was therefore decided to regard any mastitis occurrences within 31 days before the next calving as connected to the upcoming lactation period. Hence, cows entering their second lactation before the first occurrence of the disease were censored at day 31 before the second calving. In addition there were random right censored cows due to culling. Some cows, most likely without a second calf, were held in lactation for a long time yielding very large observed resistance or censoring times. A pedigree file of the 245 sires along with 57 of their male ancestors was available.

The mastitis resistance can be considered as a failure time variable and is hereby denoted $T$. The associated hazard function $h(t \mid x)$ expresses the instantaneous risk of failure at $T=t$ given that no failure has occurred prior to $t$. For an individual $i$ we let $t_{i}$ represent the failure time, whereas $c_{i}$ represents the censoring time if all we know is that $t_{i}>c_{i}$. It is convenient to express the observed data on individual $i$ by $\left(y_{i}, \delta_{i}\right)$, where

$$
y_{i}= \begin{cases}t_{i}, & \text { if } t_{i} \text { is observed } \\ c_{i}, & \text { if } c_{i}<t_{i}\end{cases}
$$

and where $\delta_{i}$ is the censoring indicator taking the value 1 if the observation is uncensored and 0 otherwise. A common approach for modelling univariate 
survival data is the proportional hazards model [5]:

$$
h(t \mid \boldsymbol{x})=h_{0}(t) \exp (\boldsymbol{x} \boldsymbol{\beta})
$$

where $h_{0}(t)$ is a baseline hazard function and $\boldsymbol{\beta}$ is a vector of regression coefficients. The probability of no failure by time $t$ is given by the survival function $S(t \mid x)$. For continuous $t$ this is given as

$$
S(t \mid x)=\exp \left\{-H_{0}(t) \exp (\boldsymbol{x} \boldsymbol{\beta})\right\}
$$

where $H_{0}(t)=\int_{0}^{t} h_{0}(u) \mathrm{d} u$ is the cumulative baseline hazard function.

In many situations, it is reasonable to assume a priori some level of smoothness of the baseline hazard function. There are several suggestions on how to perform Bayesian non-parametric modelling of the baseline hazard function. These are mostly based on the assumption of a random prior process such as the gamma process [13] or the beta process [11]. We will just assume a non-informative first order smoothing prior for the log baseline hazard of the mastitis data. More precisely, the time axis is partitioned into intervals $I_{l}=\left(t_{(l-1)}, t_{(l)}\right]$ defined by the $L$ distinct time points with observed failures $t_{(l)}$ $(l=1, \ldots, L)$, where $0<t_{(1)}<t_{(2)}<\cdots<t_{(L)}<\infty$. The log baseline hazard function is assumed piecewise constant. Let $\log \left(\lambda_{l}\right)=\log \left(h_{0}(t)\right)$ for all $t \in I_{l}$. The prior density for $\lambda=\left[\lambda_{1}, \ldots, \lambda_{L}\right]$ is, up to normalisation, assumed to be equal to

$$
p(\lambda) \propto \exp \left[-\frac{1}{\tau_{\lambda}^{2}} \sum_{l=2}^{L}\left(\log \left(\lambda_{l}\right)-\log \left(\lambda_{l-1}\right)\right)^{2}\right] .
$$

This prior is improper, has no mean and induces smoothing of the posterior log-hazards where the degree of smoothing depends on the magnitude of the smoothing parameter $\tau_{\lambda}^{2}$. Every level $\lambda_{l}$ tends to be similar to its predecessor $\lambda_{l-1}$ and its successor $\lambda_{l+1}$. Gustafson, Aeschliman and Levy [7] discuss a similar model, with a smoothing performed at the level of curvature, i.e. of order two. These authors also perform inference on $\tau_{\lambda}^{2}$, while we shall fix a value $\left(\tau_{\lambda}^{2}=0.01\right)$ satisfactorily giving smoothing and perform sensitivity analysis with respect to this choice.

A phenotypic value (e.g. milk yield, weight or disease resistance) of an animal is assumed to be the result of a genetic and an environmental component. Variation across animals with regards to the phenotypic value thus reflects both the genetic variation in the population and the excess variation due to varying environmental conditions. Both genetic and environmental variables are therefore included in the regression part of the hazard (1). 
The additive genetic effects of the 245 sires, being the fathers of the 36178 cows with records, were to be predicted in this study. The vector $\boldsymbol{s}=\left[s_{1}, \ldots, s_{245}\right]$ of genetic values was assumed to be distributed as $\boldsymbol{s} \sim$ $N_{245}\left(\mathbf{0}, \sigma_{s}^{2} \boldsymbol{A}\right)=p\left(\boldsymbol{s} \mid \sigma_{s}^{2}\right)$, where $\sigma_{s}^{2}$ is the additive genetic variance and $\boldsymbol{A}$ is the additive genetic relationship matrix.

The number of heifers from each herd is limited in our data (on average 6.8). At the herd level, we expect, as is well known, a high variability due to the small number of cows per herd. Such variability is due to the small sample size and to highly variable actual herd effects. Much of such herd effects do vary significantly, but we assume that the effects of climate, environment, veterinary follow-up, herd sizes and management vary smoothly over the geography of Norway, though differences between, say, north and south Norway may be large. Herds belonging to the same district experience the same veterinary treatment policy, since they are all under supervision of the same district veterinarian. The average number of herds per district in our data was 27 . It is reasonable to assume some smoothing at the herd level. We model such smoothing by means of aggregating herd effects within veterinary district effects and spatially smoothing at the latter level. This is similar to smoothing at the herd level, but it incorporates explicitly the "hard" information about boundaries between veterinary districts. It would be interesting, though, to include both smoothed veterinary district and herd effects, but this is left to a future study.

In Norway there are 200 veterinary districts each consisting of 1-10 municipalities. A spatial prior is assumed for the district effects on mastitis resistance. Because these effects include smooth climatic and environmental factors and similar veterinary habits, a smooth surface can be assumed a priori. Regional meetings between district veterinarians are held, which may be a source to regional similarities, hence adjacent veterinary districts can be assumed to experience a similar district effect. Let $v_{j}$ represent the effect of district $j$ (for $j=1, \ldots 200)$ and let $v$ be the $(200 \times 1)$-vector of these. The prior assumed for $v$ in the analysis was

$$
p(v) \propto \exp \left[-\frac{1}{\tau_{v}^{2}} \sum_{j=1}^{200} \sum_{j^{\prime}}\left(v_{j^{\prime}}-v_{j}\right)^{2}\right]
$$

where the summing index $j^{\prime}$ adj $j$ indicates all districts $j^{\prime}$ sharing a border with district $j$. This is again a standard improper smoothing prior, the strength of which depends on the parameter $\tau_{v}^{2}$, which we fixed as $\tau_{v}^{2}=0.1$. This value gives a balanced level of a posteriori smoothing and again sensitivity analysis 
is performed. As mentioned before, the estimation of such smoothing parameters is a difficult task. Bayesian cross-validation or direct estimation are possible but computationally demanding. We performed sensitivity analysis and found that the interpretation of our results was robust with respect to the level of smoothing within a reasonable range.

In addition to the sire and district effects, the effect of year of first calving (1990, 1991 or 1992), the effect of calving season (winter, spring, summer or autumn) and the effect of the age of the cow (in months) at first calving, were included in the regression model. This introduces eight additional parameters, denoted by $\gamma_{k}(k=1,2,3), \eta_{m}(m=1,2,3,4)$ and $\alpha$, respectively.

\section{ESTIMATION}

In the following paragraph we assume that censored observations tied with observed failures occur immediately after these, and a censoring in the interval $\left(t_{(l-1)}, t_{(l)}\right]$ occurs at $t_{(l-1)}$ (as in Breslow [3]). Let $\beta$ comprise the age effect $\alpha$, the year effects $\gamma$, the season effects $\boldsymbol{\eta}$ and the district effects $\boldsymbol{v}$. Related cows are conditionally independent given $\boldsymbol{s}$. The likelihood given the data $\boldsymbol{y}=\left\{y_{i}\right\}$ and $\delta=\left\{\delta_{i}\right\}$ (for $i=1, \ldots, n$ ) conditional on $\boldsymbol{s}$ is

$$
\begin{aligned}
& p(\boldsymbol{y}, \boldsymbol{\delta} \mid \boldsymbol{\beta}, \boldsymbol{\lambda}, \boldsymbol{s})=\prod_{l=1}^{L} \lambda_{l}^{f_{l}} \prod_{i=1}^{n} \exp \left(\boldsymbol{x}_{i} \boldsymbol{\beta}\right.\left.+s_{(i)}\right)^{\delta_{i}} \\
& \times \prod_{i=1}^{n} \exp \left\{-\exp \left(\boldsymbol{x}_{i} \boldsymbol{\beta}+s_{(i)}\right) \sum_{l: t(l) \leq y_{i}} \Lambda_{l}\right\}
\end{aligned}
$$

where $f_{l}$ is the number of failures at time $t_{(l)}, \boldsymbol{x}_{i}$ is the covariate vector of individual $i$ and $\Lambda_{l}=\lambda_{l}\left(t_{(l)}-t_{(l-1)}\right)$.

Let $p(\alpha), p(\boldsymbol{\gamma}), p(\boldsymbol{\eta})$ and $p\left(\sigma_{s}^{2}\right)$ denote hyperprior distributions for $\alpha, \boldsymbol{\gamma}, \boldsymbol{\eta}$ and for the sire variance, respectively. The joint posterior distribution for $\boldsymbol{\beta}, \lambda$, $s$ and $\sigma_{s}^{2}$ is up to proportionality given by

$$
p\left(\boldsymbol{\beta}, \lambda, \boldsymbol{s}, \sigma_{s}^{2} \mid \boldsymbol{y}, \boldsymbol{\delta}\right) \propto p(\boldsymbol{y}, \boldsymbol{\delta} \mid \boldsymbol{\beta}, \boldsymbol{\lambda}, \boldsymbol{s}) p\left(s \mid \sigma_{s}^{2}\right) p\left(\sigma_{s}^{2}\right) p(\boldsymbol{\beta}) p(\lambda)
$$

where $p(\boldsymbol{\beta})=p(\alpha) p(\boldsymbol{\gamma}) p(\boldsymbol{\eta})$. Inference from (6) is performed by means of Markov chain Monte Carlo. Specifically for our analysis, we assumed rather non-informative hyperpriors: for the inverse of $\sigma_{s}^{2}$ a gamma prior distribution with shape and scale parameters equal to 0.001 was chosen. For all other parameters, normal priors with mean zero and variance 1000 were assumed. 


\section{SIRE RANKING}

A first way to rank sires is by comparing posterior means of the sire effects. Such posterior means are the Bayesian counterpart to the BLUP estimates [8] in a non-Bayesian linear setting. Posterior means are optimal in the sense that they minimise the posterior Bayesian risk for a quadratic loss function. However, it is difficult to say whether there really is a difference between the sires based on the posterior means. We suggest a further criterion: we computed the probability $P^{a}$ that each sire in turn is among the $a \%$ best ones and then use this probability to rank sires. We believe that comparing such probabilities, that a sire is (say) among the $10 \%$ best ones, is intuitive and easier to interpret correctly than a more abstract posterior mean effect. From $P^{a}$ one might observe that a group of sires are more or less equal, yet superior to the rest. There is also a second important argument: while means are computed from univariate marginal densities, the probability $P^{a}$ is based on the full 245-dimensional joint distribution of all sire effects and hence reveals known and unknown dependencies.

One advantage of the MCMC based Bayesian approach is that these probabilities, which cannot be expressed analytically, can easily be derived from the MCMC-iterates of $s$ [2]. Say we want to estimate the requested probability for sire number 1 . Let $s_{1, b}$ be the value of $s_{1}$ at iteration $b(b=1, \ldots B)$ and $\operatorname{rank}\left(s_{1, b}\right)$ the ranking of this genetic value among the corresponding values of all sires. The estimated probability is given by

$$
P_{1}^{a}=\frac{1}{B} \sum_{b=1}^{B} I\left(\operatorname{rank}\left(s_{1, b}\right) \leq \frac{a}{100} \cdot n_{s}\right)
$$

where $I(\cdot)$ is the indicator function taking the value one if its argument is true and zero otherwise, and $n_{s}$ is the number of sires. The posterior probability is approximated simply with the fraction of the iterates for which the genetic value of the sire is among the $a \%$ best.

\section{RESULTS}

A random walk Metropolis-Hastings $(\mathrm{MH})$ algorithm was implemented. Normal or uniform proposal distributions were used in the $\mathrm{MH}$-algorithm, and these were tuned to give an acceptance probability between 0.2 and 0.5 . A total of 100000 iterations were run after burn-in from which every 10th iterate was saved yielding chains of length 10000 as the basis for statistical inference. 

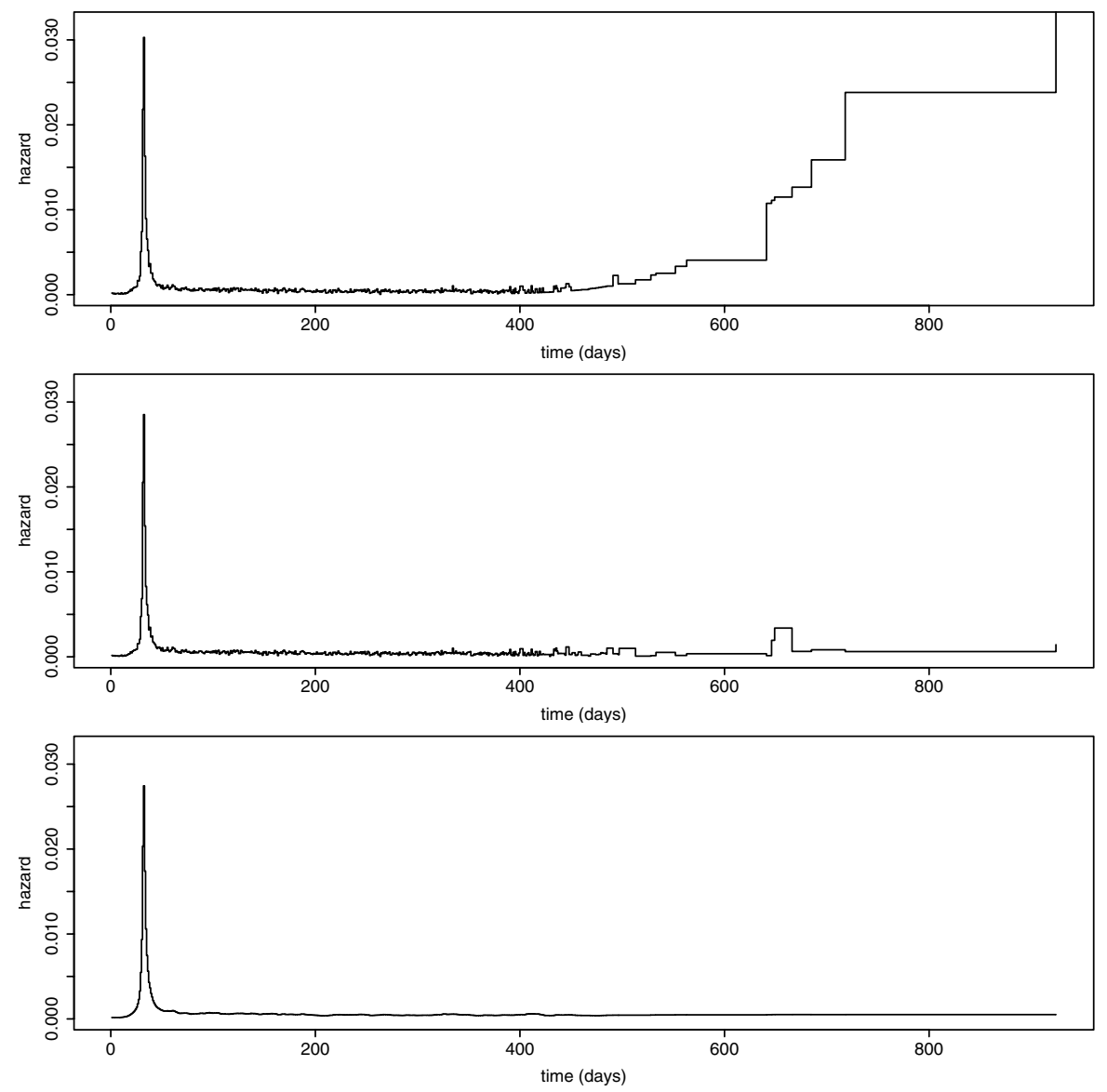

Figure 1. A non-parametric estimate of the hazard function (top) and the posterior estimates of the baseline hazard for $\tau_{\lambda}^{2}=100$ (center) and for $\tau_{\lambda}^{2}=0.01$ (bottom).

Two independent chains were run with starting values equal to the state at iteration 30000 of the main chain, and convergence was stated on the basis of negligible divergence between the chains.

The lower panel of Figure 1 shows the posterior mean estimate of the baseline hazard function for $\tau_{\lambda}^{2}=0.01$. The autoregressive structure of the prior for the baseline gave a smooth function compared to the non-parametric estimate (upper panel). The effect of smoothing is especially apparent for large values of $T$ where the small amount of data gives highly uncertain estimates without smoothing. The baseline hazard is fairly constant throughout the entire period except in the days of calving where the risk of mastitis seems highly elevated. This sudden increase in risk can be explained by physiological changes in the 
Table I. Posterior mean estimates of the regression parameter for the age effect, the year effects, the season effects and the sire variance component. In addition estimates of the standard deviation and the $2.5 \%$ - and $97.5 \%$-percentiles of the posterior distribution are given.

\begin{tabular}{lllll}
\hline Parameter & Mean & St. dev & $2.5 \%$ & $97.5 \%$ \\
\hline$\alpha$ (age) & $1.6 \times 10^{-4}$ & $1.4 \times 10^{-4}$ & $-1.1 \times 10^{-4}$ & $4.3 \times 10^{-4}$ \\
$\gamma_{1}(1990)$ & -0.040 & 0.024 & -0.086 & 0.0057 \\
$\gamma_{2}(1991)$ & $8.1 \times 10^{-4}$ & 0.015 & -0.028 & 0.030 \\
$\gamma_{3}(1992)$ & 0.040 & 0.025 & 0.0097 & 0.089 \\
$\eta_{1}$ (winter) & -0.0093 & 0.020 & -0.049 & 0.030 \\
$\eta_{2}$ (spring) & 0.036 & 0.024 & -0.012 & 0.083 \\
$\eta_{3}$ (summer) & 0.0074 & 0.018 & -0.028 & 0.043 \\
$\eta_{4}$ (autumn) & -0.035 & 0.019 & -0.072 & 0.0026 \\
$\sigma_{s}^{2}$ (sire variance) & 0.057 & 0.0095 & 0.041 & 0.078 \\
\hline
\end{tabular}

immune system as well as in the mammary glands of the cow [14]. Figure 1 also shows the degree of smoothing for $\tau_{\lambda}^{2}=100$. The posterior estimate of the baseline did not seem to be very sensitive to the prior choice of $\tau_{\lambda}^{2}$. For the posterior estimates of the other variables included in the model the choice of this smoothing parameter had only minor effects.

Some summary results from the regression analysis are given in Table I. The analysis revealed no clear effect of the age of the cow at calving, but there seemed to be an increased risk of mastitis over the years 1990-1992. This increase was in accordance with the observed increase in mastitis frequency over these years [9]. Although not as clear, there seemed to be some effect of calving season. The risk of disease was higher for spring calving cows than for cows calving in the autumn months.

The results showed a clear difference between the sires with the highest and smallest genetic value. The estimated relative risk for the sire with the smallest genetic value was 0.61 (symmetric $95 \%$ credible interval $[0.45,0.82]$ ), while the sire with the largest value had a relative risk of 1.41 ([1.12, 1.75]). This indicates a $40 \%$ decrease in hazard for daughters of the most favourable sire compared to an average sire, and an equal $40 \%$ increase in hazard for daughters of the least favourable sire.

Table II contains the 10 top-ranked sires in measure of estimated posterior means. For each, the estimated probabilities of being the best sire and of being among the $10 \%$ and $20 \%$ best sires are also given. There is a substantial drop 
Table II. The ten best sires in measure of estimated posterior means, and for each the probabilities of being the best, among the $10 \%$ best and among the $20 \%$ best.

\begin{tabular}{cccccc}
\hline Sire & Rank & $\hat{E}(s \mid \boldsymbol{y}, \boldsymbol{\delta})$ & $\operatorname{Pr}($ best $)$ & $\operatorname{Pr}(\in 10 \%$ best $)$ & $\operatorname{Pr}(\in 20 \%$ best $)$ \\
\hline 149 & 1 & -0.502 & 0.15 & 0.86 & 0.95 \\
79 & 2 & -0.478 & 0.14 & 0.80 & 0.94 \\
77 & 3 & -0.453 & 0.07 & 0.78 & 0.91 \\
104 & 4 & -0.426 & 0.06 & 0.69 & 0.88 \\
182 & 5 & -0.418 & 0.08 & 0.65 & 0.82 \\
102 & 6 & -0.393 & 0.04 & 0.60 & 0.83 \\
178 & 7 & -0.363 & 0.05 & 0.50 & 0.73 \\
236 & 8 & -0.363 & 0.04 & 0.52 & 0.75 \\
212 & 9 & -0.358 & 0.02 & 0.50 & 0.76 \\
238 & 10 & -0.351 & 0.02 & 0.47 & 0.71 \\
\hline
\end{tabular}

in the computed probabilities of being among the $10 \%$ or $20 \%$ best sires as we move down Table II, and for the least favorable sire (sire 125) the probability of being among the $20 \%$ best was found to be zero.

Sire 149 had an estimated probability of 0.15 of being the best sire. Furthermore, for sire 79 the corresponding probability was 0.14 , while for the next sire (sire 182) the probability was 0.08 . Hence, sires 149 and 79 stand out as more or less equally superior sires. Such information of subgroups of preferable sires cannot be read out of the posterior means.

As can be seen from Table II the choice of $a$ has some influence on the sire ranks, but we observe that re-ranking occurred mainly between sires with small differences in probabilities. For these sires, selecting one above the other should not be very critical. The choice of $a$ should therefore not be very crucial, but a value similar to the planned fraction of sires to be selected seems reasonable.

As expected there seemed to be a large environmental effect to the risk of mastitis. The mean relative risks for the two most extreme veterinary districts were 0.64 and 1.54 with symmetric $95 \%$ credible intervals $[0.53,0.75]$ and $[1.08,2.18]$ respectively. Recall that the time variable was time to first treatment of mastitis. These differences between veterinary districts could therefore be explained by varying treatment schemes. A low risk could reflect a higher reluctance among veterinarians against treatment initiation, or lower eager among farmers to report light infections, whereas it may be tradition for more immediate actions whenever the disease is discovered and reported 
in other regions. This may partly be true, but is probably not the full explanation. A geographical factor also seemed to be highly influential, as can be seen below. The probability of each veterinary district to be among the $a \%$ with the lowest risk of mastitis can be computed using the analogue to equation (7). Figure 2 shows these probabilities for $a=10$ plotted according to geographic location for four different values of the spatial smoothing parameter $\tau_{v}^{2}$. For $\tau_{v}^{2}=0.01$ there is a strong smoothing across veterinary districts, whereas for $\tau_{v}^{2}=10$ there is almost no smoothing effect at all. It is quite apparent from these plots that geographic location is important. Even for a low level of smoothing, the south-eastern parts of Norway seem to stand out as regions with a low relative risk of mastitis, and this impression becomes increasingly apparent as the level of smoothing goes up. Environmental factors common to the south-eastern districts appear to lower the risk of mastitis. One such factor may be that the dairy cattle managements in this region are few in number, since the south-eastern parts of Norway are among the best arable areas in the country. However, the few managements are probably large with long traditions in milk production.

Although the value of $\tau_{v}^{2}$ seems to be important for the spatial smoothing, the posterior estimates of the other parameters were not very sensitive to the prior choice of this smoothing parameter. Only small fluctuations were detected for the posterior means for the regression parameters and the sire variance. Fortunately, the ranking of sires based on predicted effects was not very affected by the level of spatial smoothing, as well. This can be seen from Figure 3 (lower triangular), where pairwise comparisons of the predicted sire-effects for the various values of $\tau_{v}^{2}$ are shown. Also the probabilities of being among the $10 \%$ best were not very affected by the smoothing as shown in the upper triangle of the same figure. More or less the same set of sires would be selected regardless of the value of $\tau_{v}^{2}$. For instance, by comparing the groups of the 25 most favorable sires for $\tau_{v}^{2}=10$ and $\tau_{v}^{2}=0.01$ respectively, we found an overlap of 23 sires (Fig. 4).

\section{DISCUSSION}

The Bayesian approach to survival analysis has in recent years become more feasible due to the advances in computer intensive statistical methods. A good presentation of Bayesian survival analysis theory and applications can be found in Ibrahim et al. [12]. The use of this methodology in conjunction with animal breeding has also gained increased attention lately. Important contributions in that respect are Ducrocq and Casella [6] and Korsgaard et al. [15]. However, 


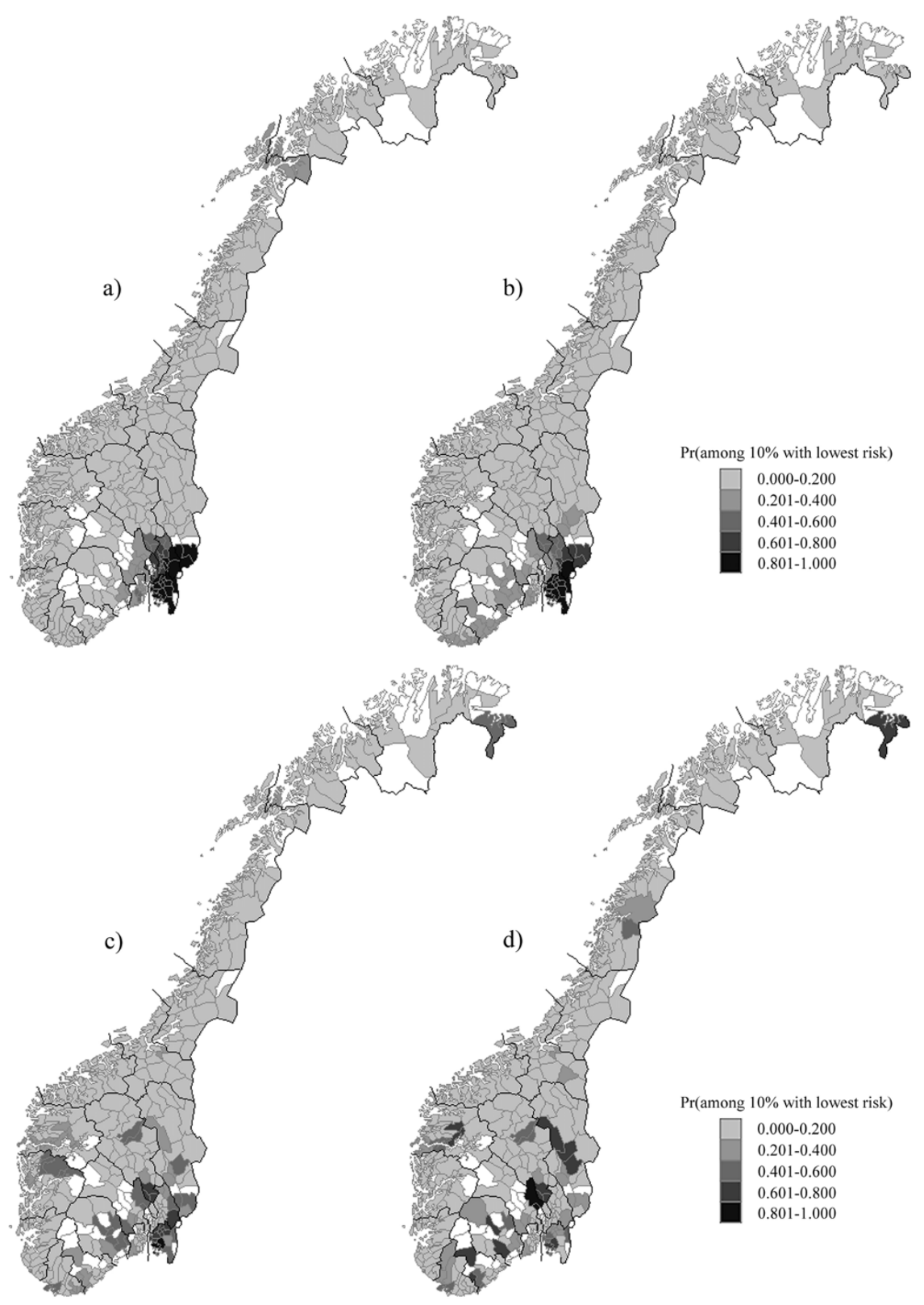

Figure 2. Geographical distribution of the probabilities of being among the $10 \%$ best districts with regards to risk of mastitis for different values of $\tau_{v}^{2}$ : a) 0.01, b) 0.1 , c) 1 and d) 10. The borders of the map correspond to municipalities, but all municipalities belonging to the same veterinary district share the same value. White regions are municipalities without data in the analysis. 

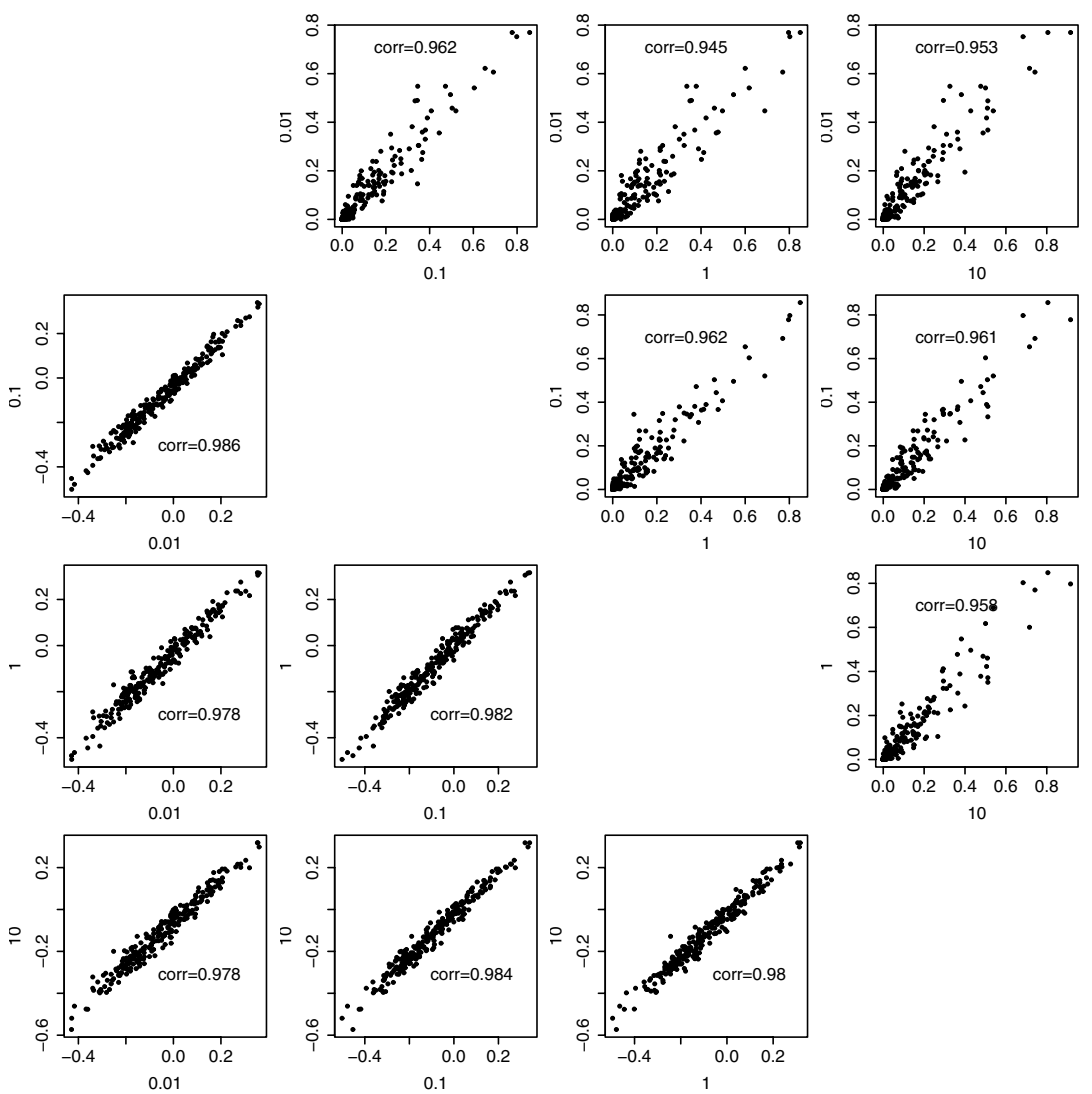

Figure 3. Upper triangle: Pairwise comparisons of the sire specific probabilities of being among the $10 \%$ best sires for different values of $\tau_{v}^{2}$ (the values are printed on the axes). Lower triangle: Pairwise comparisons of the predicted sire effects for different values of $\tau_{v}^{2}$.

with regards to the analysis of mastitis, the binary response approach using threshold models has sofar been dominating $[4,9,10]$.

Mastitis is a disease whose occurrence is highly correlated with milk yield. Cows with high milk production are likely to have more infections and mastitis than low-yield cows. In order to identify those sires who have daughters which show high mastitis resistance in addition to being good producers, milk yield should probably be included as a covariate in the model, as well. Most likely, there are also other variables which should be included in the analysis.

We did not address the question of heritability in this paper, nor did we discuss the sire variance component. The latter is not very informative unless related to the total variability. Defining a measure for heritability for survival 


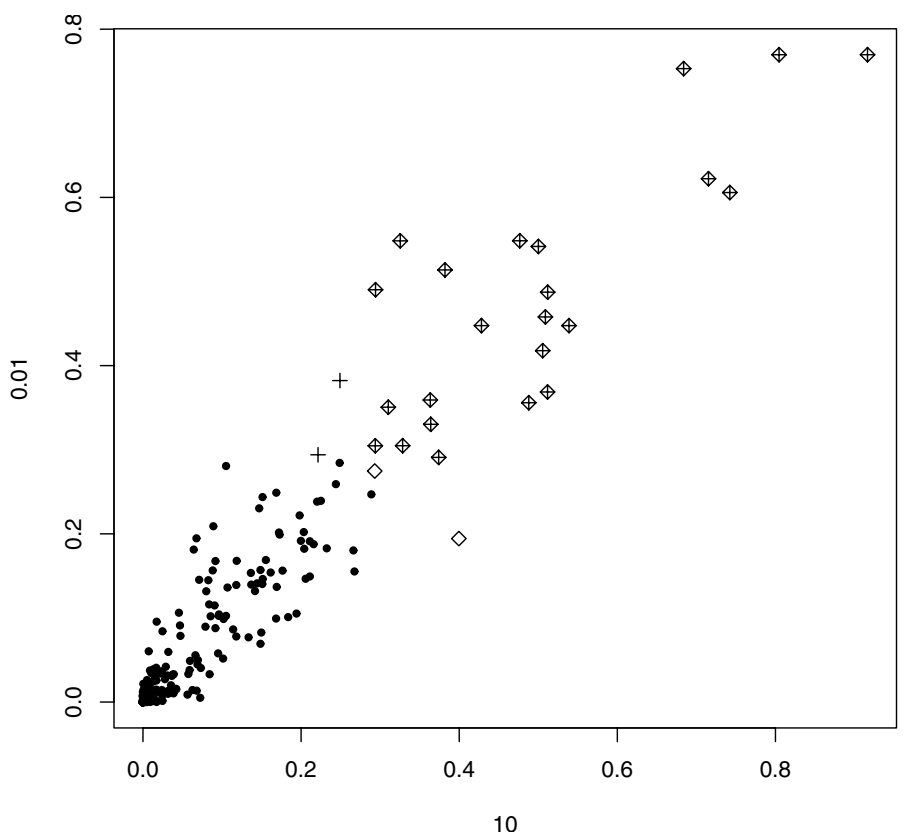

Figure 4. Comparisons of the sire specific probabilities of being among the $10 \%$ best sires for two values of $\tau_{v}^{2}$. The 25 most favourable sires are marked with + for $\tau_{v}^{2}=$ 0.01 and with $\diamond$ for $\tau_{v}^{2}=10$, and 23 sires belong to the top group in both cases.

traits is not straight forward, although some suggestions have been given on the log-time scale for proportional hazards models $[16,20]$. However, it was not the scope of this paper to contribute in that direction.

The Bayesian approach with MCMC facilitates the computation of posterior probabilities of complex events, as illustrated in this paper by sire specific probabilities of being among the best sires. We believe that the potential of this deserves more attention in many areas of applied statistical research.

\section{ACKNOWLEDGEMENTS}

This work has partly been financed by the Norwegian Research Council through the project Healthy Cow - Dairy Cattle Records - A Tool for Improved Animal Health. The Norwegian Dairy Herd Recording (Husdyrkontrollen) is acknowledged for providing data, and GENO Breeding and A.I. Association for sire pedigree information. We would also like to give credit to project SIP 154079-420. Finally we want to thank the editors and the reviewers for their valuable comments on our manuscript. 


\section{REFERENCES}

[1] Barkema H.W., Schukken Y.H., Lam T.J., Beiboer M.L., Benedictus G., Brand A., Management practices associated with the incidence rate of clinical mastitis, J. Dairy Sci. 82 (1999) 1643-1654.

[2] Besag J., Higdon D., Bayesian analysis of agricultural field experiments, J. R. Statist. Soc. B 61 (1999) 691-746.

[3] Breslow N.E., Covariance analysis of censored survival data, Biometrics 30 (1974) 89-99.

[4] Chang Y.M., Gianola D., Heringstad B., Klemetsdal G., Correlations between clinical mastitis in different periods of first-lactation Norwegian Cattle using a multivariate threshold model, in: Gatsonis C., Carriquiry A., Gelman A., Higdon D., Kass R., Pauler D., Verdinelli I. (Eds.), Case studies in Bayesian statistics, volume VI, Springer-Verlag, New York, 2002.

[5] Cox D.R., Regression models and life-tables (with discussion), J. R. Statist. Soc. B 34 (1972) 187-220.

[6] Ducrocq V., Casella G., A Bayesian analysis of mixed survival models, Genet. Sel. Evol. 28 (1996) 505-529.

[7] Gustafson P., Aeschliman D., Levy A.R., A simple approach to fitting Bayesian survival models, Lifetime Data Anal. 9 (2003) 5-19.

[8] Henderson C.R., Prediction of future records, in: Pollack E., Kempthorne O., Bailey T.B. (Eds.), Proc. Int. Conf. Quantitative Genetics, Iowa State Univ., Ames, Iowa, USA, 1977, pp. 615-638.

[9] Heringstad B., Klemetsdal G., Ruane J., Clinical mastitis in Norwegian cattle: frequency, variance components, and genetic correlation with protein yield, J. Dairy Sci. 82 (1999) 1325-1330.

[10] Heringstad B., Chang Y.M., Gianola D., Klemetsdal G., Genetic analysis of longitudinal trajectory of clinical mastitis in first lactation Norwegian Cattle, J. Dairy Sci. 86 (2003) 2676-2683.

[11] Hjort N.L., Nonparametric Bayes estimators based on beta processes in models for life history data, Ann. Statist. 18 (1990) 1259-1294.

[12] Ibrahim J.G., Chen M.H., Sinha D., Bayesian Survival Analysis, SpringerVerlag, New York, 2001.

[13] Kalbfleisch J.D., Nonparametric Bayesian analysis of survival time data, J. R. Statist. Soc. B 40 (1978) 214-221.

[14] Kehrli M.E., Nonnecke B.J., Roth J.A., Immunosuppression in dairy cows at calving, Am. J. Vet. Res. 50 (1989) 207-214.

[15] Korsgaard I.R., Madsen P., Jensen J., Bayesian inference in the semiparametric log normal frailty model using Gibbs sampling, Genet. Sel. Evol. 30 (1998) 241-256.

[16] Korsgaard I.R., Andersen A.H., Jensen J., Discussion of heritability of survival traits, in: Proc. GIFT, Jouy-en-Josas, France, Interbull Bulletin No. 21 (1999) $31-35$.

[17] Morse D., Lorenzo M.A., Wilcox C.J., Collier R.J., Natzko R.P., Bray D.R., Climatic effects on occurrence of clinical mastitis, J. Dairy Sci. 71 (1988) 848-853. 
[18] Schreiner D.A, Ruegg P.L., Relationship between udder and leg hygiene scores and subclinical mastitis, J. Dairy Sci. 86 (2003) 3460-3465.

[19] Sorensen D.A., Andersen D., Gianola D., Korsgaard I.R., Bayesian inference in threshold models using Gibbs sampling, Genet. Sel. Evol. 27 (1995) 229-249.

[20] Yazdi M.H., Visscher P.M., Ducrocq V., Thompson R., Genetic parameters and response to selection in proportional hazards models, in: Book of Abstracts of the 51st Annual Meeting of the European Association for Animal Production, The Netherlands, Wageningen Pers, Book of Abstracts No. 6 (2000) 81.

To access this journal online: www.edpsciences.org 